\title{
Zinc Coordination to Multiple Ligand Atoms in Organic-rich Surface Soils
}

\author{
Carmen Enid Martínez ${ }^{1, *}$, Katya A. Bazilevskaya ${ }^{1}$, and Antonio Lanzirotti ${ }^{2}$
}

${ }^{1}$ Department of Crop and Soil Sciences, The Pennsylvania State University, University Park, PA

${ }^{2}$ Consortium for Advanced Radiation Sources, The University of Chicago and National Synchrotron Light Source, Brookhaven National Laboratory, NY

\section{Supporting Information.}

Table S1 presents the stability constants for $\mathrm{Zn}, \mathrm{Cu}$, and $\mathrm{Ni}$ with ligands containing O-, N-, and S- functional groups. Figure S1 shows synchrotron-based x-ray analyses for the soil labeled Manning 3 (M3). 
Table S1. Stability Constants (expressed as Log K values) for Various Metal-Ligand Complexes. ${ }^{1,2}$

\begin{tabular}{|c|c|c|c|c|}
\hline Ligand & Equilibrium & $\mathbf{Z n}$ & $\mathbf{C u}$ & $\mathbf{N i}$ \\
\hline \multirow[t]{2}{*}{ Nitrate $\left(\mathrm{NO}_{3}{ }^{-}\right)$} & $\mathrm{ML} / \mathrm{M} \cdot \mathrm{L}$ & 0.4 & 0.5 & 0.4 \\
\hline & $\mathrm{ML}_{2} / \mathrm{M} \cdot \mathrm{L}^{2}$ & -0.3 & -0.4 & -0.5 \\
\hline \multirow[t]{2}{*}{ Acetate $\left(\mathrm{CH}_{3} \mathrm{COOH}\right)$} & $\mathrm{ML} / \mathrm{M} \cdot \mathrm{L}$ & 1.6 & $1.87 / 2.2$ & 1.4 \\
\hline & $\mathrm{ML}_{2} / \mathrm{M} \cdot \mathrm{L}^{2}$ & 1.8 & 3.6 & \\
\hline \multirow[t]{2}{*}{ Citrate $\left(\mathrm{HOOCCH}_{2}-(\mathrm{C}(\mathrm{OH}) \mathrm{COOH})-\mathrm{CH}_{2} \mathrm{COOH}\right)$} & $\mathrm{ML} / \mathrm{M} \cdot \mathrm{L}$ & 6.1 & 7.2 & 6.7 \\
\hline & $\mathrm{ML}_{2} / \mathrm{M} \cdot \mathrm{L}^{2}$ & 6.8 & & \\
\hline Glycine $\left(\mathrm{NH}_{2}-\mathrm{CH}_{2}-\mathrm{COOH}\right)$ & $\mathrm{ML} / \mathrm{M} \cdot \mathrm{L}$ & 5.16 & 8.22 & 6.10 \\
\hline Aspartic acid ( $\left.\mathrm{HOOC}-\mathrm{CH}_{2}-\mathrm{CH}\left(\mathrm{NH}_{2}\right)-\mathrm{COOH}\right)$ & $\mathrm{ML} / \mathrm{M} \cdot \mathrm{L}$ & $5.84,2.90^{3}$ & 8.57 & 7.12 \\
\hline Glutamic acid $\left(\mathrm{HOOC}-\left(\mathrm{CH}_{2}\right)_{2}-\mathrm{CH}\left(\mathrm{NH}_{2}\right)-\mathrm{COOH}\right)$ & $\mathrm{ML} / \mathrm{M} \cdot \mathrm{L}$ & 5.45 & 7.85 & 5.90 \\
\hline \multirow[t]{2}{*}{ Cysteine (HS- $\left.\mathrm{CH}_{2}-\mathrm{CH}\left(\mathrm{NH}_{2}\right)-\mathrm{COOH}\right)$} & $\mathrm{ML} / \mathrm{M} \cdot \mathrm{L}$ & $9.17 / 11.0$ & gets reduced & 9.82 \\
\hline & $\mathrm{ML}_{2} / \mathrm{M} \cdot \mathrm{L}^{2}$ & $18.18 / 19.80$ & & 20.07 \\
\hline \multirow[t]{2}{*}{ Methionine $\left(\mathrm{CH}_{3}-\mathrm{S}-\mathrm{CH}_{2}-\mathrm{CH}_{2}-\mathrm{CH}\left(\mathrm{NH}_{2}\right)-\mathrm{COOH}\right)$} & $\mathrm{ML} / \mathrm{M} \cdot \mathrm{L}$ & 4.37 & 7.87 & 5.19 \\
\hline & $\mathrm{ML}_{2} / \mathrm{M} \cdot \mathrm{L}^{2}$ & 8.33 & 14.72 & 9.84 \\
\hline \multirow[t]{2}{*}{ Lysine $\left(\mathrm{NH}_{2}-\left(\mathrm{CH}_{2}\right)_{4}-\mathrm{CH}\left(\mathrm{NH}_{2}\right)-\mathrm{COOH}\right)$} & $\mathrm{ML} / \mathrm{M} \cdot \mathrm{L}$ & & 7.56 & 5.47 \\
\hline & $\mathrm{ML}_{2} / \mathrm{M} \cdot \mathrm{L}^{2}$ & 8.40 & 14.02 & 9.00 \\
\hline \multirow[t]{3}{*}{ Glutamine $\left(\mathrm{NH}_{2}-(\mathrm{C}=\mathrm{O})-\left(\mathrm{CH}_{2}\right)_{2}-\mathrm{CH}\left(\mathrm{NH}_{2}\right)-\mathrm{COOH}\right)$} & $\mathrm{ML} / \mathrm{M} \cdot \mathrm{L}$ & 4.83 & $7.75 / 8.33$ & 5.16 \\
\hline & $\mathrm{ML}_{2} / \mathrm{M} \cdot \mathrm{L}^{2}$ & 9.17 & $14.23 / 15.50$ & 9.42 \\
\hline & $\mathrm{ML}_{3} / \mathrm{M} \cdot \mathrm{L}^{3}$ & 11.84 & & \\
\hline \multirow[t]{2}{*}{ Arginine $\left(\mathrm{NH}_{2}-(\mathrm{C}=\mathrm{NH})-\mathrm{HN}-\left(\mathrm{CH}_{2}\right)_{3}-\mathrm{CH}\left(\mathrm{NH}_{2}\right)-\mathrm{COOH}\right)$} & $\mathrm{ML} / \mathrm{M} \cdot \mathrm{L}$ & 4.70 & 8.10 & 5.18 \\
\hline & $\mathrm{ML}_{2} / \mathrm{M} \cdot \mathrm{L}^{2}$ & 9.00 & 14.90 & 9.49 \\
\hline \multirow[t]{2}{*}{ Histidine $\left(\mathrm{C}_{3} \mathrm{~N}_{2} \mathrm{H}_{3}-\mathrm{CH}_{2}-\mathrm{CH}\left(\mathrm{NH}_{2}\right)-\mathrm{COOH}\right)$} & $\mathrm{ML} / \mathrm{M} \cdot \mathrm{L}$ & 6.55 & 10.20 & 8.67 \\
\hline & $\mathrm{ML}_{2} / \mathrm{M} \cdot \mathrm{L}^{2}$ & 12.06 & 18.10 & 15.54 \\
\hline
\end{tabular}

${ }^{1}$ Constants were compiled from Martell and Smith (1), Kotrlý and Šuocha (2), Stumm and Morgan (3), Stevenson (4), Buffle (5), and Furia (6).

${ }^{2}$ The constants were not determined at the same ionic strength $(I)$ or under the same experimental conditions and are therefore not strictly comparable; variations over a Log unit can be expected.

${ }^{3} \log \mathrm{K}_{(\mathrm{Zn})}=2.90$ considering protonated ammonia groups. 

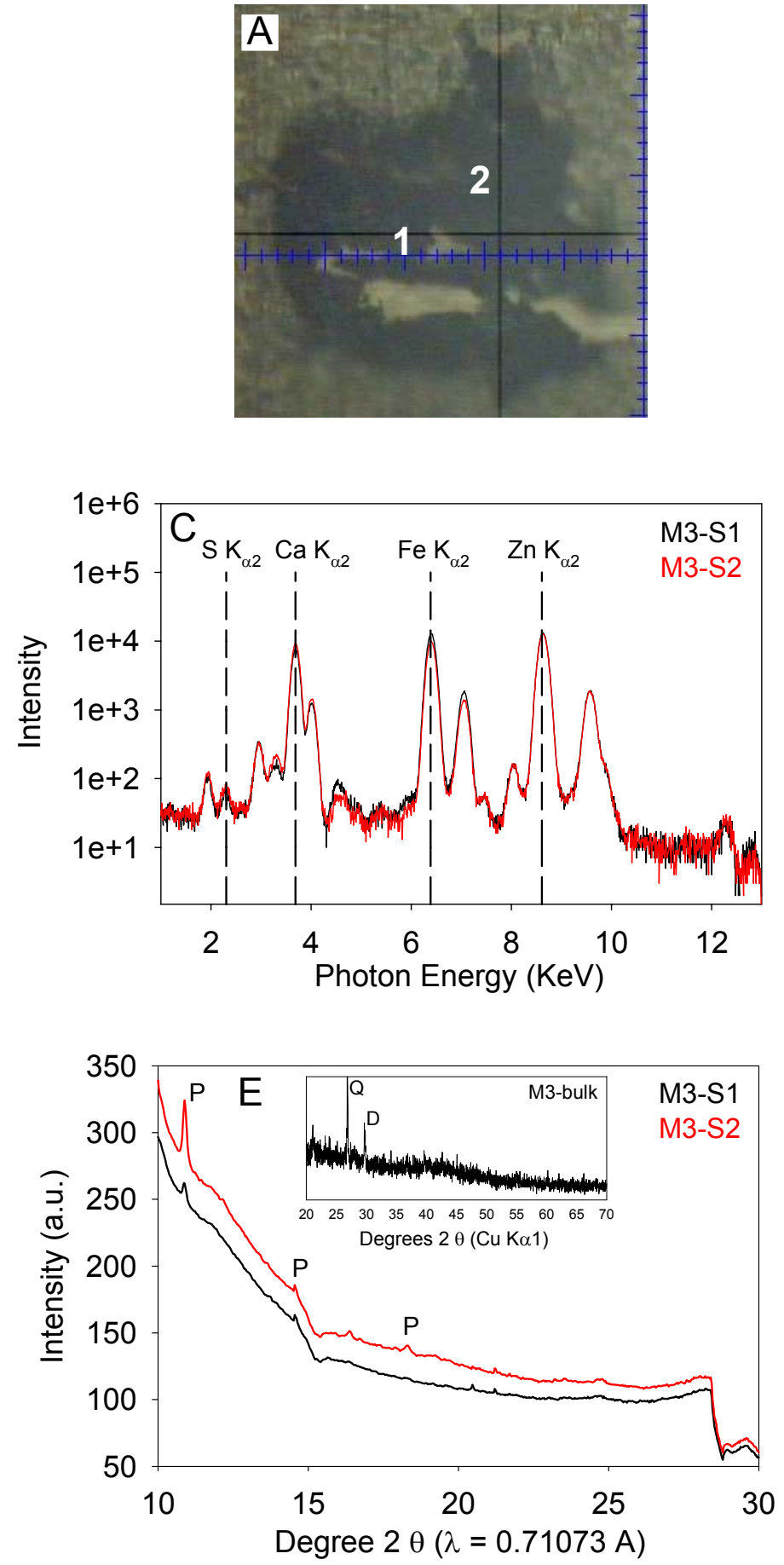
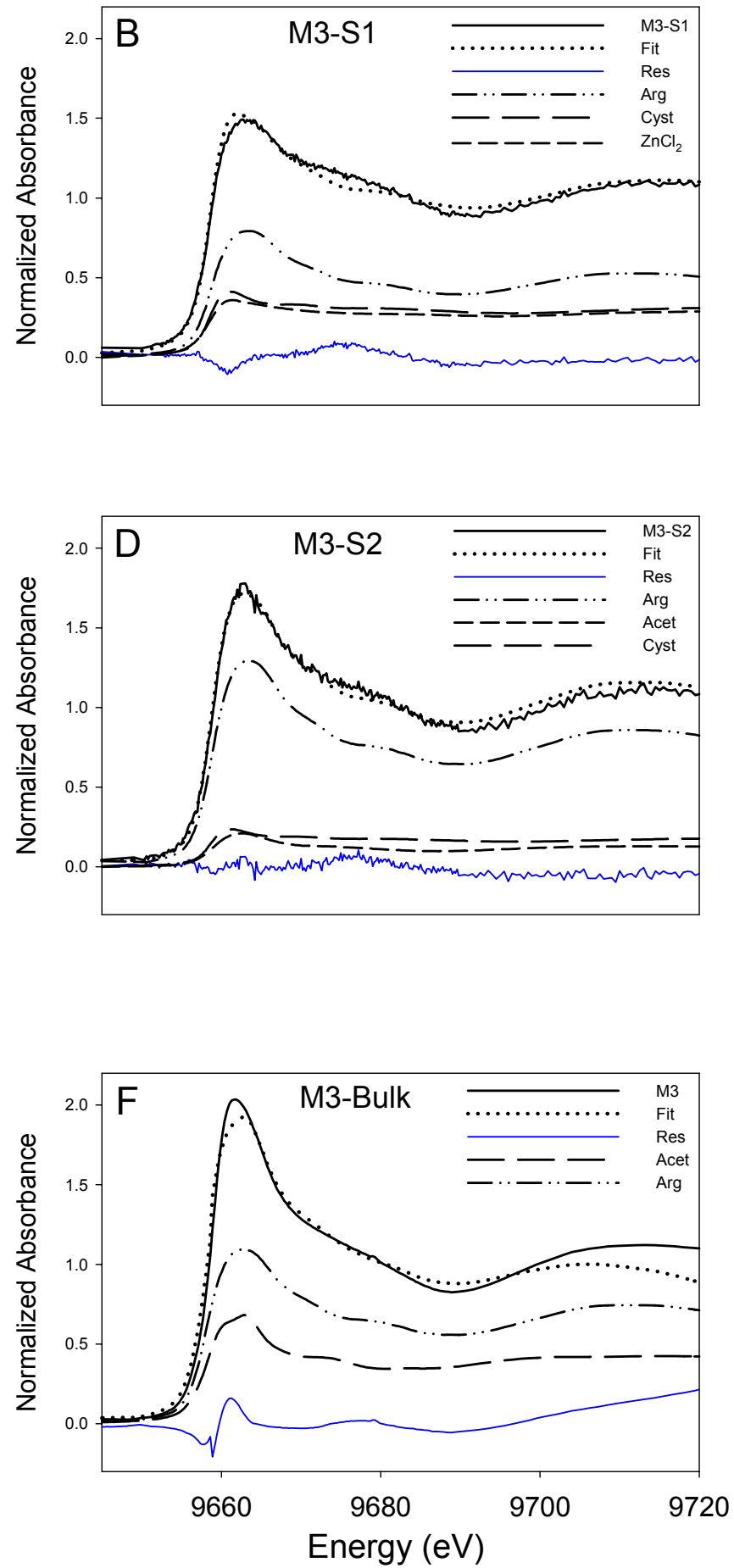

Figure S1. Synchrotron-based x-ray analyses for the soil labeled Manning 3 (M3). Panel A shows a soil particle and two $14 \times 10 \mu \mathrm{m}$ regions labeled 1 and 2 . Panel C shows the $\mu$-XRF spectra of the two regions, M3-S1 and M3-S2, while Panel E shows their two-dimensional $\mu$-XRD patterns (the XRD of the bulk soil is shown as an insert in Panel E). Also shown are the results from Linear Combination Fit (LCF) analyses of the two $10 \mathrm{x} 14$ $\mu \mathrm{m}$ regions (Panels B and D) and of the bulk soil M3 (Panel F). 


\section{References Cited}

(1) Martell, A.E.; Smith, R.M. Critical Stability Constants, Vol. 1: Amino Acids, Plenum Press, New York, p. 47-50, 1974.

(2) Kotrlý, S.; Šůcha, L. Handbook of Chemical Equilibria in Analytical Chemistry, John Wiley \& Sons, New York, p. 116-133, 1985.

(3) Stumm, W.; Morgan, J.J. Aquatic Chemistry: Chemical Equilibria and Rates in Natural Waters, $3^{\text {rd }}$ ed.; John Wiley \& Sons: New York, 1996.

(4) Stevenson, F.J. Stability constants of $\mathrm{Cu}^{2+}, \mathrm{Pb}^{2+}$, and $\mathrm{Cd}^{2+}$ complexes with humic acids. Soil Sci. Soc. Am. J. 1976, 40, 665-672.

(5) Buffle, J. Complexation reactions in aquatic systems. An analytical approach; Ellis Horwood Limited: England, 1988.

(6) Furia, T.E. CRC handbook of Food Additives, $2^{\text {nd }}$ ed.; 1972. 